\title{
El Enlace Covalente
}

\author{
Juliana Cárdenas Castaño \\ Universidad Surcolombiana, Colombia \\ julianacardenas09@gmail.com \\ Luis Javier Narváez Zamora \\ Universidad Surcolombiana, Colombia \\ ljnz47@usco.edu.co
}

\section{Introducción}

Este trabajo tiene el propósito de realizar una conceptualización sobreel proceso dehibridación o mezcla de orbitales atómicos pertenecientes a los subniveles de energía $s$ (small) y $p$ (pound) para tratar de explicar la naturaleza del enlace covalente desde la perspectiva de los modelos de la teoría del orbital molecular, del electrónvalencia y de la repulsión del par electrónico de la capa de valencia. El análisis va dirigido a definir el enlace químico, concepto de profunda aplicación tanto para estudiosos de la Química, como para entender la organización estructural de las sustancias químicas covalentes, constitutivas del universo en general.

El enlace químico es la fuerza necesaria para mantener dos o más átomos unidos formando compuestos moleculares o cristales iónicos, cualquiera sea el caso, la diferencia entre los valores de electronegatividad de dos átomos comprometidos en un enlace permite establecer el tipo de enlace establecido entre ellos, inicialmente. Pauling (1959, p. 235) planteó el valor 1.9 para asignar cerca del $50 \%$ de carácter iónico de un enlace; sin embargo, con el descubrimientoeinvención denuevas sustancias, esta diferencia se ha venido reduciendo hasta 1.6, cifra con la cual se establecen los conceptos involucrados en este artículo. Los valores para el carácter iónico de un enlace se muestran en las tablas No. 1 y tabla No. 2
Tabla No. 1. Carácter iónico porcentual de un enlace simple.

\begin{tabular}{lcccccccccccccccc}
\hline $\begin{array}{l}\text { Diferencia de } \\
\text { electronegatividad }\end{array}$ & 0.1 & 0.2 & 0.3 & 0.4 & 0.5 & 0.6 & 0.7 & 0.8 & 0.9 & 1.0 & 1.1 & 1.2 & 1.3 & 1.4 & 1.5 & 1.6 \\
\hline $\begin{array}{l}\text { \% de carácter } \\
\text { iónico }\end{array}$ & 0.5 & 1.0 & 2.0 & 4.0 & 8.0 & 9.0 & 12 & 15 & 19 & 22 & 28 & 30 & 34 & 39 & 43 & 47 \\
\hline & & & & & & & & & & & & & & & & \\
\hline $\begin{array}{l}\text { Diferencia de } \\
\text { electronegatividad }\end{array}$ & 1. & 1. & 1. & 2. & 2. & 2. & 2. & 2. & 2. & 2. & 2. & 2. & 2. & 3. & 3. & 3. \\
& 7 & 8 & 9 & 0 & 1 & 2 & 3 & 4 & 5 & 6 & 7 & 8 & 9 & 0 & 1 & 2 \\
\hline $\begin{array}{l}\text { \% de carácter } \\
\text { iónico }\end{array}$ & 51 & 55 & 59 & 63 & 67 & 70 & 74 & 78 & 79 & 82 & 84 & 86 & 88 & 89 & 91 & 92 \\
\hline
\end{tabular}

Fuente: Welch, 2014.

Tabla No. 2. Carácter iónico parcial de enlaces y diferencia de electronegatividad de átomos.

\begin{tabular}{lcccccccccccccc}
\hline $\mathrm{Xa}-\mathrm{Xb}$ & 0 & 0.2 & 0.4 & 0.6 & 0.8 & 1.0 & 1.2 & 1.4 & 1.6 & $1-8$ & 2.0 & 2.2 & 2.4 & 2.6 \\
\hline $\begin{array}{l}\text { Carácter } \\
\text { iónico } \\
\text { parcial }\end{array}$ & 0 & 1 & 3 & 7 & 12 & 18 & 25 & 32 & 40 & 47 & 54 & 61 & 68 & 74 \\
\end{tabular}

Fuente: Pauling, 1959, p. 235.

Para abordar el enlace covalente es preciso definirlo previamente: un enlace covalente ocurre cuando un par de electrones son compartidos por dos átomos. En ese sentido, Chang (2010, p. 374) lo define como "un enlace en el que dos electrones son compartidos por dos átomos". Por su parte Pauling (1959, p. 212), lo define como la propiedad que une a dos átomos, es decir, "un par compartido de electrones". En términos de Brown et al. (2004, p. 275), se define dicho enlace como aquellos que "se comparten uno o más pares de electrones entre átomos, según sea necesario para lograr un octeto de electrones en torno a cada átomo".

Para definir el enlace covalente indefectiblemente debe abordarse el concepto de hibridación de orbitales atómicos, cuya 
interacción genera los orbitales moleculares propios de las moléculas covalentes; en ese sentido, es pertinente hacer una precisión importante, es decir que en los enlaces covalentes de moléculas con octeto están comprometidos los orbitales propios correspondientes a los subniveles $\mathrm{s}$ y $\mathrm{p}$ del último nivel de energía. Aquellas moléculas con octeto aumentado o expandido, además de los 4 orbitales mencionados anteriormente, involucran uno o dos orbitales del subnivel $\boldsymbol{d}$ (dark), tal como se describe más adelante.

A continuación, se describen las interacciones de los electrones presentes en los subniveles mencionados atrás, para constituir el traslape o apantallamiento electromagnético, responsable de los orbitales híbridos.

\section{Orbitales Híbridos}

Un orbital atómico es un campo electromagnético donde existe la probabilidad de encontrar como máximo un par de electrones con spin contrario; los orbitales híbridos $\mathrm{u}$ orbitales moleculares resultan de la mezcla de orbitales atómicos comprometidos en un enlace, para el caso de moléculas sencillas, el traslape o mezcla de orbitales resulta una opción explicativa eficiente, sin embargo, para moléculas poliatómicas, los orbitales atómicos se deben mezclar formando orbitales híbridos, cuyas disposiciones espaciales son diferentes a las de los orbitales atómicos, así son posibles varios tipos de hibridación, a saber: $s p, s p^{2}, s p^{3}$, $s p^{3} d^{1}, s p^{3} d^{2}$, propias de las estructuras mostradas en la tabla No. 3 .

Tabla No. 3. Posibles geometrías moleculares de orbitales moleculares de 2 a 5 orbitales moleculares híbridos

\begin{tabular}{|c|c|c|c|c|c|c|}
\hline Hibridación & $\begin{array}{c}\text { Orbitales } \\
\text { híbridos }\end{array}$ & Geometría & $\begin{array}{c}\text { Dominios } \\
\text { enlazantes }\end{array}$ & $\begin{array}{c}\text { Dominios } \\
\text { antienlazantes }\end{array}$ & $\begin{array}{c}\text { Geometría } \\
\text { molecular }\end{array}$ & Ejemplos \\
\hline$s p$ & 2 & $\begin{array}{c}\text { Lineal } \\
s\end{array}$ & 2 & 0 & $\mathrm{~B}-\mathrm{A}-\mathrm{B}$ & $\mathrm{N} \equiv \mathrm{N}$ \\
$\mathrm{BF} 2, \mathrm{HgCl}_{2}$ \\
\hline
\end{tabular}

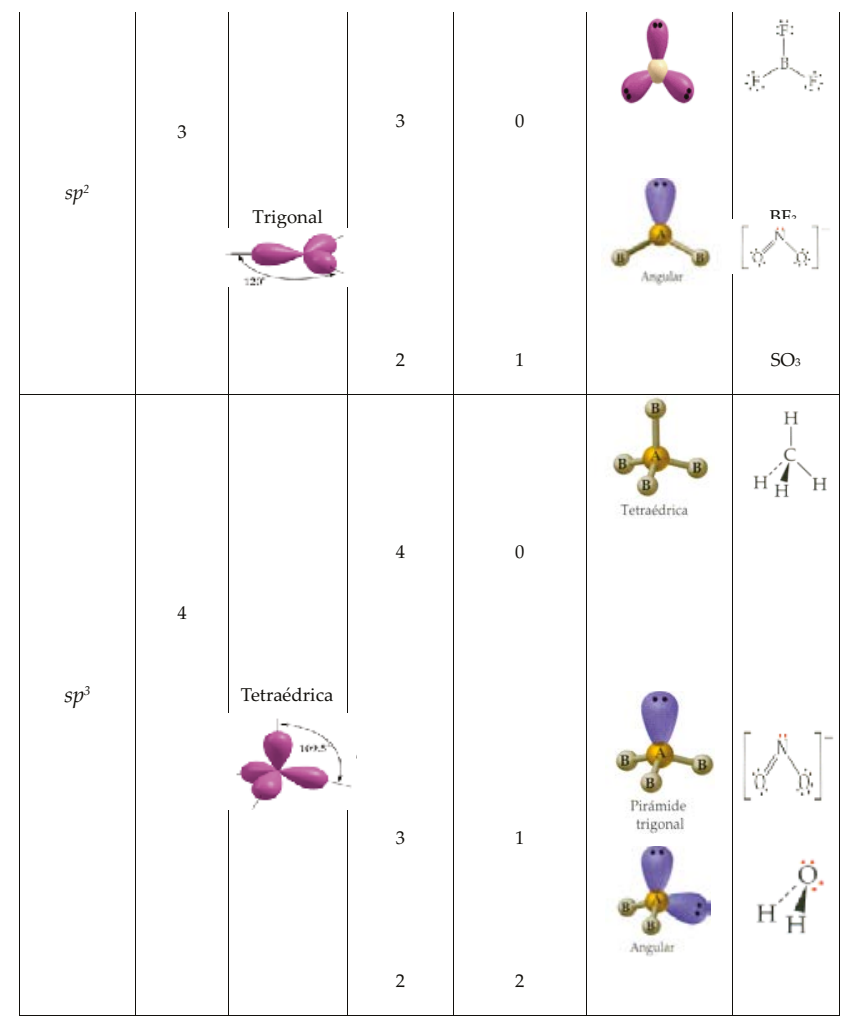

Fuente: Brown, LeMay, Bursten y Burdge. (2004, 324)

Los tipos de hibridación enunciados en la tabla No. 3 requieren un análisis más detallado. En todos los casos, la mezcla, traslape o apantallamiento de orbitales atómicos para formar orbitales moleculares, ocurre de dos maneras bien definidas: todos los orbitales comprometidos en el enlace se traslapan o, al combinarse, quedan uno o más orbitales libres sin llegar a hibridarse. La interacción o traslape de orbitales híbridos explica la formación de enlaces sigma y la interacción electrostática de orbitales no híbridos produce enlaces pi. En cada caso la explicación se aborda a partir de un ejemplo específico, donde además se describen las estructuras de Lewis, explicativas del concepto enlace.

\section{Hibridación $s p$}

Esta hibridación se explicita con una molécula sencilla, en este caso $\mathrm{N}_{2}$. Para determinar la estructura de esta molécula se inicia ubicando al $\mathrm{N}$ en el grupo $15(5 \mathrm{~A})$ y una electronegatividad de 3.0; al efectuar la resta se tiene: $3.0-3.0=0$ $<1.6$, esta diferencia de acuerdo con el umbral 
de Pauling determina el carácter covalente del enlace, con esta clasificación se elaboran las estructuras de Lewis para explicitar el tipo de enlace covalente establecido, tal como se muestra en las gráficas $1 \mathrm{a}$ y $1 \mathrm{~b}$.

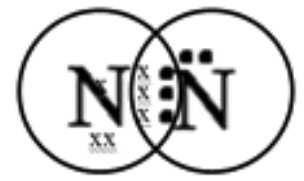

Gráfica 1a. Estructura de puntos.

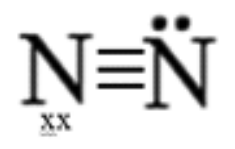

Gráfica 1b. Estructura de guiones.
Las dos estructuras determinan el requerimiento de hibridación sp para la molécula N2, tal como se muestra en la gráfica No. 2.

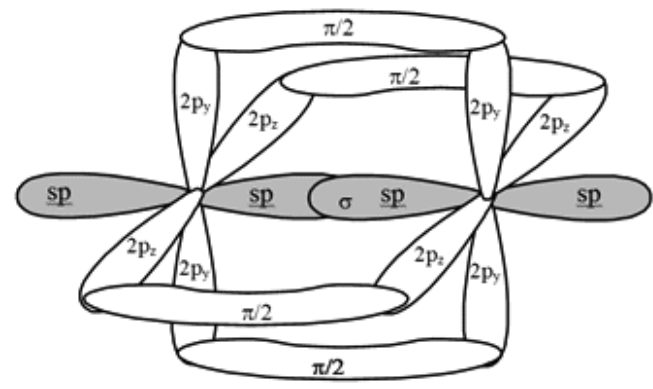

Gráfica No. 2. Orbitales moleculares de $\mathrm{N}_{2}$.

Esta molécula también se puede representar a través del modelado molecular obtenido del simulador Chem Sketch, aplicación de ACD Labs ${ }^{1}$ (Advanced Chemistry Development).

De igual forma, la molécula de nitrógeno se puede representar usando el visor en tres dimensiones de la misma aplicación anterior, ese modelo se presenta en la gráfica No. 3.

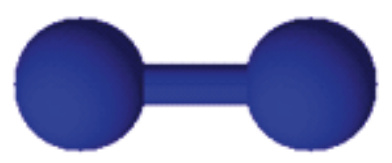

Gráfica No. 3. Modelo tridimensional de $\mathrm{N}_{2}$.

Es evidente la formación de un enlace covalente triple entre los dos átomos de nitrógeno, este enlace se explica a través de hibridación $s p$ con un ángulo de enlace de $180^{\circ}$; este enlace covalente triple en realidad y tal como se muestra en la gráfica No. 2, se encuentra formado por una interacción $\sigma$ y dos interacciones $\pi$ la interacción $\sigma$ como producto del solapamiento o traslape de dos orbitales híbridos, y las dos interacciones $\pi$ formadas por el traslape de los orbitales no híbridos $2 P_{y}$ y $2 P_{z}$.

El modelo RPECV (Repulsión del Par Electrónico de la Capa de Valencia) permite definirle a la molécula de nitrógeno, dos enlaces idénticos orientados linealmente; cada átomo de $\mathrm{N}\left(1 s^{2}, 2 s^{2}, 2 p^{3}\right)$ tiene cinco electrones en la capa de valencia, además tiene tres electrones $2 p$ desapareados en cada uno de los orbitales $2 p_{x^{\prime}} 2 p_{y}$ y $2 p_{z^{\prime}}$ los cuales pueden enlazarse con cada uno de los electrones promovidos del otro átomo excitado de nitrógeno, el cual recurre a esta promoción energética para poder enlazarse, porque en el estado basal solamente es imposible un enlace; ambos electrones de valencia se encuentran apareados como par antienlazante, la promoción o excitación antes mencionada le permite a uno de los dos electrones del orbital $2 s$, saltar a uno de los tres orbitales $2 p$ y en esas condiciones enlazarse al otro átomo de nitrógeno. Las configuraciones basal (estado neutro) y excitada del átomo de nitrógeno se muestran en la gráfica No. 4.

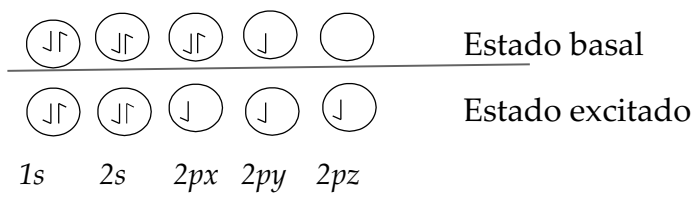

Gráfica No. 4. Estados basal y excitado para el átomo de nitrógeno.

En síntesis, para la ocurrencia del enlace covalente triple entre los dos átomos, se han mezclado o hibridado dos orbitales; el $2 s$ y el $2 p x$ del nivel de valencia, generando dos orbitales $s p$ más grandes a los orbitales iniciales, Brown et al, (2004, p. 332), proponen la definición de enlace covalente triple como "un acomodo lineal de dominios de electrones implica la hibridación sp", tal hibridación se ilustra en la gráfica No. 5 .

$1 \mathrm{http}: / / w w w . a c d l a b s . c o m / a c c o u n t / r e g i s t e r . p h p ?$ redirect=/resources/freeware/download.php 


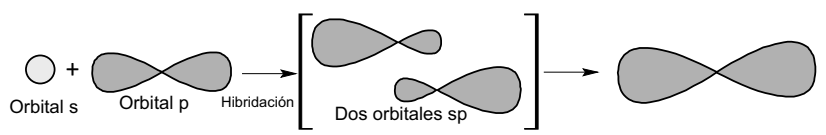

Gráfica No. 5. Hibridación $s p$.

Una vez enlazados los dos átomos de $\mathrm{N}$ y surtida la hibridación $s p$ mencionada, la estructura molecular de esta sustancia puede representarse tal como lo ilustra la gráfica No. 2 .

En la tabla No. 5 se sintetizan los aspectos más importantes de la molécula $\mathrm{N}_{2}$.

Tabla No. 5. Características de la molécula $\mathrm{N}_{2}$.

\begin{tabular}{llllllll}
\hline Átomo & $\begin{array}{l}\mathrm{e}- \\
\text { valencia }\end{array}$ & Hibridación & $\begin{array}{l}\text { Ángulo } \\
\text { de } \\
\text { enlace }\end{array}$ & $\sigma$ & $\pi$ & $\begin{array}{l}\text { Orbitales } \\
\text { enlazantes }\end{array}$ & $\begin{array}{l}\text { Orbitales } \\
\text { antienlazantes }\end{array}$ \\
\hline $\mathrm{N}^{1}$ & 5 & $s p$ & $180^{\circ}$ & 1 & 2 & 3 & 1 \\
$\mathrm{~N}^{2}$ & 5 & $s p$ & $180^{\circ}$ & 1 & 2 & 3 & 1 \\
\hline
\end{tabular}

La carga formal como algoritmo para aceptar los diagramas de Lewis construidos para estructuras covalentes se evalúa en la tabla No. 6 .

Tabla No. 6. Carga formal de la molécula de $\mathrm{N}_{2}$.

\begin{tabular}{|c|c|c|c|c|c|c|}
\hline Átomo & e- de valencia & - & $1 / 2$ e-compartidos & - & e- $\sin$ compartir & Total \\
\hline $\mathrm{N}^{1}$ & 5 & & 3 & & 2 & 0 \\
\hline \multirow[t]{2}{*}{$\mathrm{N}^{2}$} & 5 & & 3 & & 2 & 0 \\
\hline & & & & & Total & 0 \\
\hline
\end{tabular}

Si la carga formal total tiene un valor diferente a cero, la estructura molecular fue mal construida y amerita su revisión exhaustiva.

\section{Hibridación $s p^{2}$}

Hemos escogido la molécula de oxígeno para explicitar este tipo de hibridación, en la cual se mezclan tres orbitales: uno de tipo $s(2 s)$ y dos de tipo $p$ (2py y $2 p z$ ). El número atómico del oxígeno es ocho, por tal razón su configuración electrónica en el estado excitado se ilustra en la gráfica No. 6.
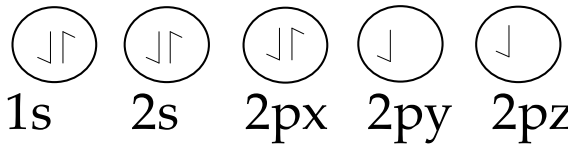

$$
1 \mathrm{~s}
$$

Gráfica No. 6. Estado excitado del átomo de oxígeno.

En este estado, cada átomo de oxígeno tiene la posibilidad de formar un enlace covalente doble con otro símil. La resta de la electronegatividad $3.5-3.5=0<1.6$ confirma el tipo de enlace antes anunciado. Solo resta elaborar las estructuras de Lewis correspondientes, las cuales se muestran en las gráficas $7 \mathrm{a}$ y $7 \mathrm{~b}$.

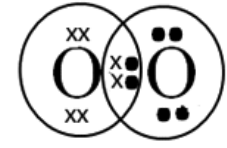

Gráfica 7a. Estructura de puntos.

\section{$\mathrm{O}=\mathrm{O}$}

Gráfica $7 \mathrm{~b}$. Estructuras de Lewis del $\mathrm{O}_{2}$.
La hibridación $s p^{2}$ correspondiente a esta molécula se indica en la gráfica No. 8 .

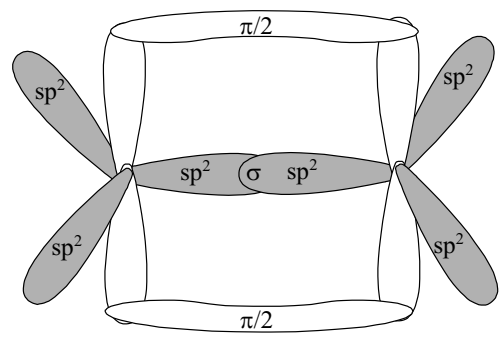

Gráfica No. 8. Hibridación $s p^{2}$ para la molécula de $\mathrm{O}_{2}$.

Esta estructura puede representarse también usando el simulador Chem Sketch como en la gráfica No. 9.

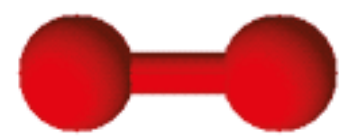

Gráfica No. 9. Modelo tridimensional de la molécula de $\mathrm{O}_{2}$.

En la tabla No. 7 se sintetizan los aspectos más importantes de la molécula $\mathrm{O}_{2}$.

Tabla No. 7. Características de la molécula $O_{2}$.

\begin{tabular}{llllllll}
\hline Átomo & $\begin{array}{l}\mathrm{e}^{-} \\
\text {valencia }\end{array}$ & Hibridación & $\begin{array}{l}\text { Ángulo } \\
\text { de } \\
\text { enlace }\end{array}$ & $\sigma$ & $\pi$ & $\begin{array}{l}\text { Orbitales } \\
\text { enlazantes }\end{array}$ & $\begin{array}{l}\text { Orbitales } \\
\text { antienlazantes }\end{array}$ \\
\hline $\mathrm{O}^{1}$ & 6 & $\mathrm{sp}^{2}$ & $120^{\circ}$ & 1 & 1 & 3 & 2 \\
$\mathrm{O}^{2}$ & 6 & $\mathrm{sp}^{2}$ & $120^{\circ}$ & 1 & 1 & 3 & 2 \\
\hline
\end{tabular}

La carga formal como algoritmo para aceptar los diagramas de Lewis construidos para estructuras covalentes se evalúa en la tabla No. 8. 
Tabla No. 8. Carga formal de $O_{2}$.

\begin{tabular}{|c|c|c|c|c|}
\hline Átomo & $\mathrm{e}^{-}$de valencia & - $\quad 1 / 2$ e compartidos & - $\mathrm{e}^{-} \sin$ compartir & Total \\
\hline $\mathrm{O}^{1}$ & 6 & 2 & 4 & 0 \\
\hline \multirow[t]{2}{*}{$\mathrm{O}^{2}$} & 6 & 2 & 4 & 0 \\
\hline & & & Total & 0 \\
\hline
\end{tabular}

En la hibridación $s p^{2}$, dos orbitales $p\left(P_{x} y\right.$ $\left.P_{4}\right)$ se hibridan con un orbital $s$, para generar 3 orbitales híbridos dispuestos en el mismo plano de simetría con ángulos de $120^{\circ}$, posición de máximo rechazo y comodidad energética. El orbital $p_{z}$ no participa de la mezcla y queda por fuera del plano de simetría de los tres orbitales híbridos $s p^{2}$, formando un ángulo de $90^{\circ}$ con este plano. Tal situación se ilustra en la gráfica No. 10.

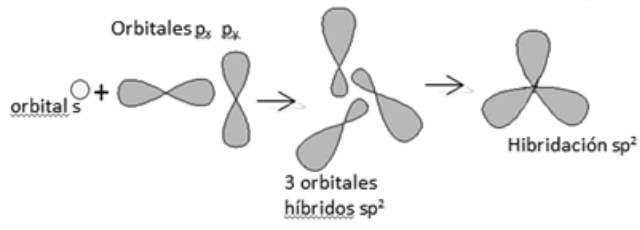

Gráfica No. 10. Hibridación $s p^{2}$.

En la gráfica 10 no se incorpora el orbital $p_{z^{\prime}}$ el cual no participa de la mezcla de orbitales, este orbital libre forma un enlace pi como ya se anunció anteriormente, en cambio, el traslape de los dos orbitales híbridos produce una interacción sigma. Un ejemplo clásico de este tipo de hibridación ocurre al interior de la molécula de eteno, la cual se ilustra en la gráfica No. 11.

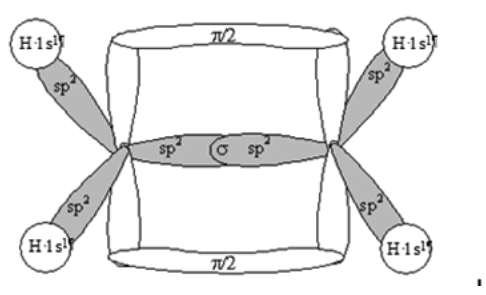

Gráfica No. 11. Hibridación $s p^{2}$ del $\mathrm{C}_{2} \mathrm{H}_{4}$.

La estructura tridimensional de la molécula se indica en la gráfica No. 12.

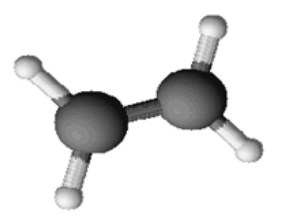

Gráfica No. 12. Estructura de la molécula del eteno.
En esta gráfica se evidencian las siguientes características: la molécula es plana como consecuencia de dos situaciones: el enlace sigma ocurrido como solapamiento de los dos orbitales híbridos de cada átomo de carbono enlazado, y por igual, el enlace pi se ubica por encima y por debajo del plano molecular $(\pi / 2$ por encima $y$ $\pi / 2$ por debajo de este plano)

\section{Hibridación $s p^{3}$}

Para la hibridación $s p^{3}$, escogimos la molécula de agua donde se mezclan tres orbitales $p\left(P_{x} . P_{y}\right.$ y $\left.P_{z}\right)$ con un orbital s del átomo de oxígeno, para generar cuatro orbitales híbridos dispuestos espacialmente de forma tetraédrica con el fin de contrarrestar el rechazo electrostático debido a la presencia de dos electrones en cada uno de ellos. El oxígeno y el hidrógeno se encuentran ubicados en los grupos $6 \mathrm{~A}$ o 16 y 1 respectivamente y los valores de sus electronegatividades son 3.5 y 2.1. La diferencia de electronegatividades 3.5-2.1=1.4<1.6 conduce a plantear la ocurrencia de un enlace covalente. Las estructuras de Lewis para la molécula de agua se indican en las gráficas 13a y $13 b$.

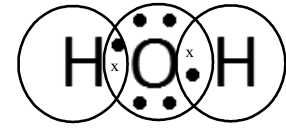

Gráfica No. 13a.

Estructura de puntos.

\section{$\mathrm{H}-\mathrm{O}-\mathrm{H}$}

Gráfica No. 13b.

Estructura de guiones.
Por igual, a través del Chem sketch la estructura tridimensional de la molécula adquiere la configuración mostrada en la gráfica No. 14.

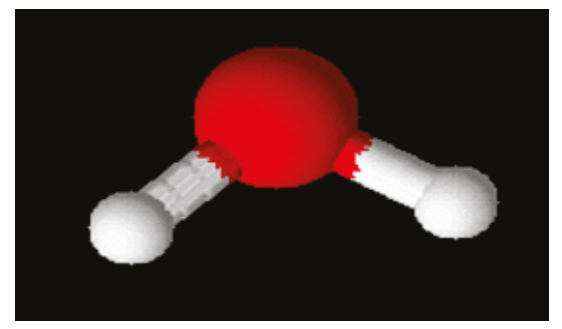

Gráfica No. 14. Estructura tridimensional de la molécula de agua.

La misma estructura desde la perspectiva de los orbitales moleculares se visualiza en la gráfica No. 15. 


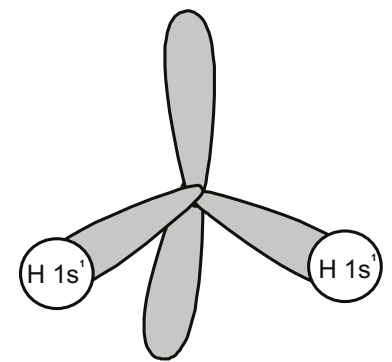

Gráfica No. 15. Orbitales moleculares para la molécula del agua.

La hibridación $s p^{3}$ es un solapamiento o mezcla de un orbital de tipo s y tres de tipo $p$, tal como se anunció anteriormente, de manera esquemáticamente en las gráficas No. 16, 17 y 18.

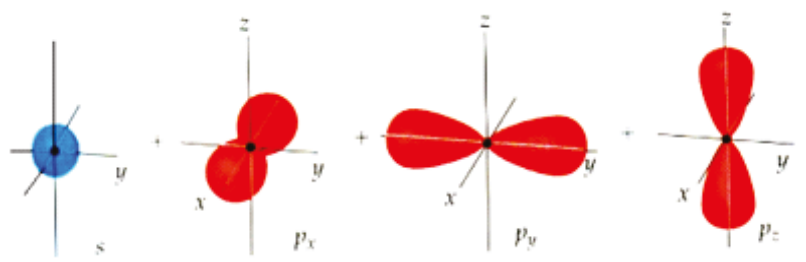

Gráfica No. 16. Orbitales participantes en de la hibridación $s p^{3}$.

Fuente: Brown, et al, $(2004,325)$.

La mezcla de los cuatro orbitales antes anunciada se denota detalladamente en la gráfica No. 17.
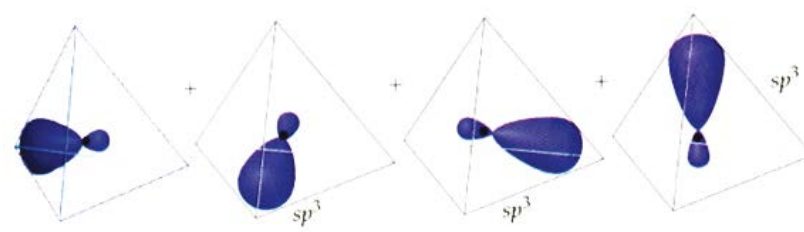

Gráfica No. 17. Detalle de la hibridación $s p^{3}$.

Fuente: Fuente: Brown, et al, $(2004,335)$.

El arreglo final se muestra en la gráfica No. 18.

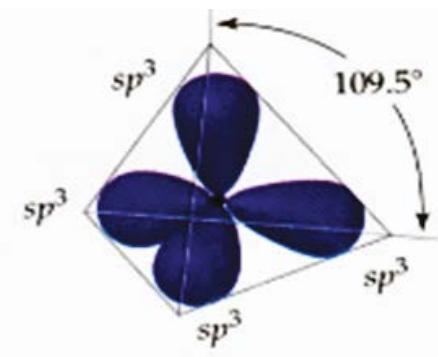

Gráfica No. 18. Disposición geométrica tetraédrica de la hibridación $s p^{3}$.

Fuente: Fuente: Brown, et al, (2004, 325).
La hibridación $s p^{3}$ produce cuatro orbitales híbridos, responsables del enlace sigma cuando se traslapan con orbitales del mismo tipo. Bajo este tipo de hibridación no son posibles los enlaces pi por carecer de orbitales libres en la mezcla electrostática.

Otros ejemplos donde se requiere la hibridación $s p^{3}$ se ilustran en los casos detallados a continuación.

En primer lugar, el alcano n-butano con fórmula $\mathrm{C}_{4} \mathrm{H}_{10}$, una vez efectuados los cálculos pertinentes para determinar el tipo de enlace y la estructura geométrica, da como resultado lo presentado en las gráficas No. 19 y 20.

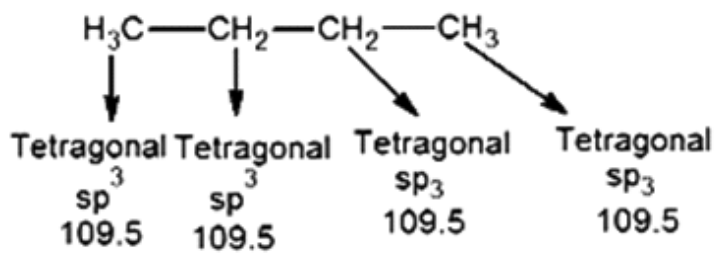

Gráfica No. 19. Tipo de hibridación del n-butano Cárdenas Castaño (2017).

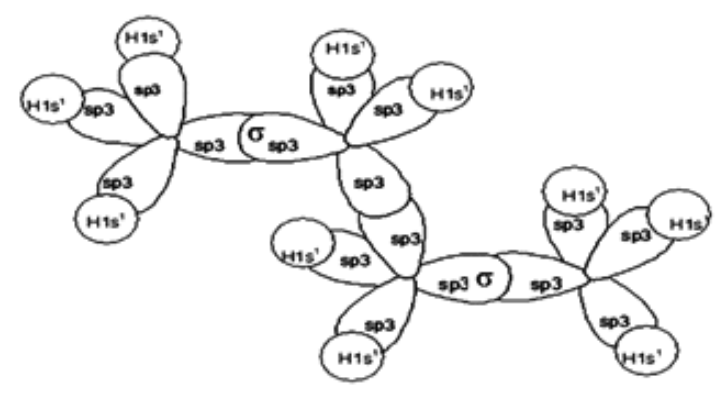

Gráfica No. 20. Orbitales moleculares de n-butano Cárdenas Castaño (2017).

Para el caso de moléculas con capas de valencia expandida, superando los lineamientos de la ley de octeto, la posibilidad de explicar este fenómeno se concreta involucrando los orbitales del subnivel $d$, situación propia de los elementos del tercer periodo, los cuales mezclan un orbital $s$ con tres de tipo p y uno o dos orbitales de tipo $d$, tal y como se presenta en Narváez Zamora (2009). 
En el primer caso se producen cinco orbitales híbridos de tipo $s p^{3} d^{1}$, en el espacio, estos cinco nuevos orbitales se dirigen a los extremos de una bipirámide trigonal, formando tres ángulos de $120^{\circ}$ y seis de $90^{\circ}$, tal cual se indica en la gráfica No. 21.

A su vez, la mezcla de un orbital s, con 3 de tipo p y dos de tipo $d$, genera seis orbitales híbridos $s p^{3} d^{2}$, los cuales se orientan hacia los vértices de un octaedro, de tipo bipiramidal cuadrada formando 12 ángulos de $90^{\circ}$, tal cual se ilustra en la gráfica No. 22. El abordaje conceptual de estos dos tipos de hibridación será abordado por los autores en una reflexión ulterior.

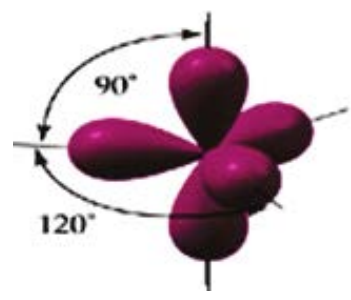

Gráfica No. 21.

Hibridación $s p^{3} d^{1}$

Fuente: Brown, LeMay, Bursten y Burdge. (2004, 336)

\section{Moléculas con hibridación mixta}

Nos hemos permitido explicitar la naturaleza del enlace covalente basados en el modelo de la hibridación de orbitales atómicos para generar orbitales moleculares, a través de los cuales es posible explicar el enlace covalente sencillo, doble, triple y coordinado o dativo. Sin embargo, las sustancias con moléculas covalentes existen también en la naturaleza con una mixtura de estos enlaces, tal como se evidencia en los casos planteados a continuación.

El alqueno 1-buteno, cuya fórmula molecular es $\mathrm{C}_{4} \mathrm{H}_{8}$ posee hibridación $s p^{3}$ y $s p^{2}$ tal como se indica en las gráficas No. 23 y No. 24.

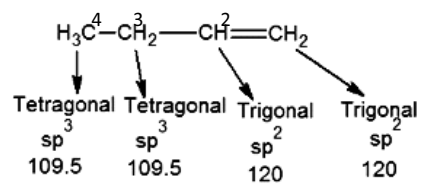

Enlace $\pi$ : $s p^{2}$ de $C_{1}$ y $s p^{2}$ de $C_{2}$ Enlace $\sigma: \mathrm{sp}^{2}$ de $\mathrm{C}_{2}$ y $\mathrm{sp}^{3}$ de $\mathrm{C}_{3}$ Enlace $\sigma: \mathrm{sp}^{3}$ de $\mathrm{C}_{3}$ y $\mathrm{sp}^{3}$ de $\mathrm{C}_{4}$

Gráfica No. 23. Hibridaciones $s p^{3}$ y $s p^{2}$ del 1-buten. Cárdenas Castaño (2017).

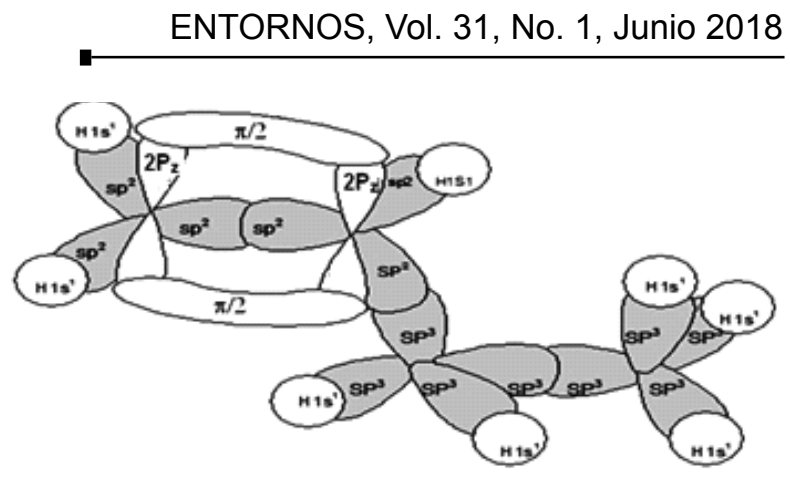

Gráfica No. 24. Orbitales moleculares del 1-buteno. Cárdenas Castaño (2017)

En cuanto a la estructura molecular del 2-butino, un alquino con hibridación $s p^{3}$ y $s p$, sus estructuras se detallan en las gráficas No. 25 y No. 26.

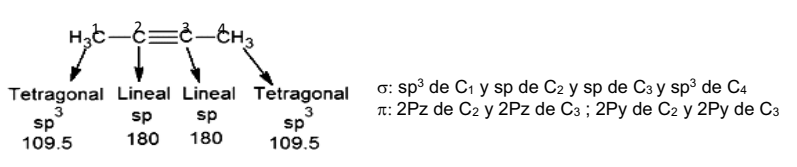

Gráfica No. 25. Hibridaciones $s p^{3}$ y $s p$ del 2-butino. Cárdenas Castaño (2017).

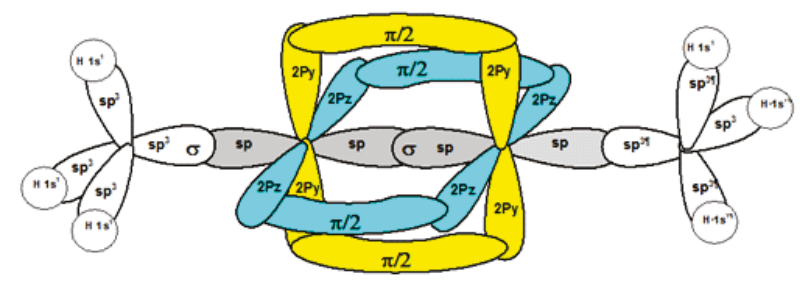

Gráfica No. 26. Orbitales moleculares del 2-butino. Cárdenas Castaño (2017).

\section{Conclusiones}

En síntesis, los autores pretenden efectuar un acercamiento conceptual hacia el mundo submicroscópico del enlace químico de tipo covalente, desde las perspectivas de las teorías de enlace vigentes a través de modelos científicos y mentales, derivados de estudios rigurosos derivados de los planteamientos de Linus Pauling sobre su percepción de la manera como se unen los átomos.

El abordaje teórico del artículo trata de demostrar cómo se unen los átomos, cuando el tipo de enlace es covalente, así mismo, y a manera de conclusión se plantea que las propiedades físicas y químicas de las sustancias químicas 
con enlace covalentes dependen exclusivamente de su estructura geométrica molecular, la cual a su vez se deriva del tipo de hibridación comprometido en el enlace.

La hibridación es un modelo para explicar los mecanismos electromagnéticos a través de los cuales, los átomos interactúan para establecer enlace covalente, el cual ocurre entre átomos con valores de electronegatividad cuya diferencia sea menor a 1.6; la hibridación en consecuencia es el resultado del apantallamiento o traslape de los orbitales atómicos correspondientes al nivel o capa de valencia, Tal interacción puede ocurrir entre orbitales híbridos para formar interacciones sigma, propias del enlace sencillo, o entre orbitales no híbridos, cuya interacción deriva en interacciones pi, propias de enlaces covalentes doble o triples.

Los tres tipos de hibridación $\left(s p, s p^{2}\right.$ y $\left.s p^{3}\right)$ abordados por los autores, tienen ángulos de enlace de $180^{\circ}, 120^{\circ}$ y $109^{\circ}$ respectivamente; estas disposiciones geométricas les confieren a las moléculas covalentes, las propiedades fisicoquímicas anunciadas anteriormente.

En próximas oportunidades, los autores abordarán el enlace iónico y el enlace metálico para involucrar átomos cuya posibilidad de enlace es diferente al covalente y así efectuar una mayor cobertura de las moléculas que hacen parte de nuestro vasto universo.

\section{Referencias bibliográficas}

ACD Labs. Advanced Chemistry Development. Consultado el 9 de octubre de 2017, disponible en: http://www.acdlabs.com/ account/register.php? redirect=/resources/ freeware/download.php

Brown, T., LeMay, E., Bursten, B., \& Burdge, J. (2004). Química, La Ciencia Central (Novena ed.). México: Pearson Prentice Hall.

Cárdenas Castaño, J. (2017). Moléculas, apuntes de clase. Neiva: Universidad Surcolombiana.

Chang, R. (2010). Química (Décima ed.). México: Mc Graw Hill.

Narváez Zamora, L. (2009). Aprendizaje de los conceptos Enlace químico y Estequiometría. Neiva: Universidad Surcolombiana.

Pauling, L. (1959). General Chemistry, An Introduction to Descriptive Chemistry and Modern Chemical Theory (Second edition ed.). San Francisco, California: W. H. Freeman and Company.

Welch, S. (2014). Tabla de las propiedades periódicas de los elementos. Chicago: Sargent Welch Scientific Company. 